\title{
Methodologies and Applications of Wireless Mobile Ad-hoc Networks Routing Protocols
}

\author{
Shiva Prakash \\ Assistant Professor, CSE \\ Deptt., Madan Mohan Malaviya \\ Engineering College, \\ Gorakhpur-273010, INDIA
}

\author{
J. P. Saini \\ Principal, Madan Mohan \\ Malaviya Engineering College \\ Gorakhpur-273010, INDIA
}

\author{
S. C. Gupta \\ P.G. Coordinator \\ Dehradun Institute of \\ Technology, Dehradun, \\ Uttarakhand, INDIA
}

\begin{abstract}
An important and essential issue for mobile ad-hoc networks (MANETs) is routing protocol design that is a major technical challenge due to the dynamism of the network. MANETs have applications in rapidly deployed and dynamic military and civilian systems. The network topology in a MANET usually changes with time. Therefore, there are new challenges for routing protocols in MANETs since traditional routing protocols may not be suitable for MANETs. During the last years, active research work resulted in a variety of proposals. This research focuses on the methodologies of different typical types of routing protocols and then compared these protocols based on common characteristics and overall comparison based on basic characteristic. After that we presented applications and real challenges of routing protocols in MANET. This paper aims to aid those MANET's researchers and application developers in selecting appropriate routing protocols for their work. Also, this paper can support formal verification of MANET routing protocols or efficient implementation of these routing protocols.
\end{abstract}

\section{Keywords}

Mobile Ad Hoc Network, Routing Protocols, Ah Hoc Applications.

\section{INTRODUCTION}

Wireless networks have become increasingly popular in the communication industry. This is particularly true with in the past decade, which has seen wireless networks being adapted to enable mobility. The mobile wireless network is the Infrastructure less mobile network, commonly known as mobile ad- hoc networks. Ad-hoc networks have no fixed routers [1] all nodes are capable of movement and can be connected dynamically in an random manner. People can deploy a wireless network easily and quickly. End users can move around while staying connected to the network. Wireless networks play an important role in both military and civilian systems [5][27]. Handheld personal computer connectivity, notebook computer connectivity, vehicle and ship networks, and rapidly deployed emergency networks are all applications of this kind of network. Hosts and routers in a wireless network can move around. Therefore, the network topology can be dynamic and unpredictable. Traditional routing protocols used for wired networks cannot be directly applied to most wireless networks because some common assumptions are not valid in this kind of dynamic network.
Routing protocols in MANETS have been proposed and previous work focused on designing new protocols, comparing existing protocols, or improving protocols before standard MANET routing protocols are defined[3][4][28]. The majority research in this field is based on simulation studies of the adhoc routing protocols of interest in arbitrary networks with certain traffic profiles. However, the simulation results from different research groups are not consistent. This is because of the lack of consistency in MANET routing protocol models and application environments including networking and user traffic profiles. Therefore, simulation scenarios used in past studies are not reasonable for all protocols and their conclusions cannot be generalized. Furthermore, this is complicated for one to choose a appropriate routing protocol for a given MANET application. However, there has been little research on this kind of framework [1][12].

As promising network type in future mobile applications, mobile ad hoc networks are attracting more and more researchers. This paper gives the characteristics, classification and fundamentals for typical routing protocols for mobile ad hoc networks [15][38], including classical MANET unicast and multicast routing algorithms and popular classification methods. In this paper, related routing protocols are compared from an analysis point of view based on the classification methods.

Rest of the paper is organized as follows. Section 2 gives classifications for mobile ad hoc routing protocols. Section 3 presents the comparisons and analysis of different routing approaches. Section 4 deals and challenges of mobile ad-hoc network routing protocols and finally section 5 concludes the paper with future work.

\section{CLASSIFICATION OF TYPICAL ROUTING PROTOCOLS}

There are different criteria for designing and classifying routing protocols for wireless ad hoc networks [2][24][26][28]. For example, what routing information is exchanged; when and how the routing information is exchanged, when and how routes are computed and so on? Some classification of ad hoc network protocols is listed below: 


\subsection{Pro-active (Table Driven) Routing}

This type of protocols maintains fresh lists of destinations and their routes by periodically distributing routing tables throughout the network. The main disadvantages of such algorithms are respective amount of data for maintenance and slow reaction on restructuring and failures.

Proactive protocols continuously evaluates the routes within the network so that when we are required to forward the packet route is already known and immediately ready for use[5][8]. So, there is no any time delay (time spend in route discovery process) takes place. So a shortest path can be find without any time delay however these protocols are not suitable for very dense ad hoc networks because in that condition problem of high traffic may arise. Several modifications of proactive protocols have been proposed for removing its shortcomings and use in ad hoc networks. It maintains the unicast routes between all pair of nodes without considering of whether all routes are actually used or not. It can be of two types depending upon the algorithms which have been shown in the next section. In link state proactive protocols each node maintains a view of the network topology and it stores the cost of each outgoing links and periodically broadcast its link costs via flooding. In distance vector proactive protocols each node maintains a routing table which contains the cost of every node of the network, next node to reach the destination and the total no of nodes to reach the destination and this routing information table is send to all neighbors' to maintain the topology. Examples of the proactive protocols are[10][12][18] - Ad-hoc Wireless Distribution Service, Clusterhead Gateway Switch Routing Protocol[21], Highly Dynamic Destination-Sequenced Distance Vector routing protocol[1][3], Hierarchical State Routing protocol[20], Intrazone Routing Protocol/pro-active part of the ZRP[35], Linked Cluster Architecture[1], Mobile Mesh Routing Protocol[4], Optimized Link State Routing Protocol[40],Topology Dissemination based on Reverse-Path Forwarding routing protocol[22][29], Witness Aided Routing[5], and Wireless Routing Protocol[27].

\subsubsection{Dynamic Destination-Sequenced Distance- Vector Routing Protocol}

The Destination-Sequenced Distance-Vector (DSDV) Routing Algorithm [3][26][15] is based on the idea of the classical Bellman-Ford Routing Algorithm with certain improvements. Every mobile station maintains a routing table that lists all available destinations, the number of hops to reach the destination and the sequence number assigned by the destination node. The sequence number is used to distinguish stale routes from new ones and thus avoid the formation of loops. The stations periodically transmit their routing tables to their immediate neighbors. A station also transmits its routing table if a significant change has occurred in its table from the last update sent. So, the update is both time-driven and eventdriven. The routing table updates can be sent in two ways - a "full dump" or an incremental update. A full dump sends the full routing table to the neighbors and could span many packets whereas in an incremental update only those entries from the routing table are sent that has a metric change since the last update and it must fit in a packet. If there is space in the incremental update packet then those entries may be included whose sequence number has changed. When the network is relatively stable, incremental updates are sent to avoid extra traffic and full dump are relatively infrequent. In a fastchanging network, incremental packets can grow big so full dumps will be more frequent. Each route update packet, in addition to the routing table information, also contains a unique sequence number assigned by the transmitter. The route labeled with the highest (i.e. most recent) sequence number is used. If two routes have the same sequence number then the route with the best metric (i.e. shortest route) is used. Based on the past history, the stations estimate the settling time of routes. The stations delay the transmission of a routing update by settling time so as to eliminate those updates that would occur if a better route were found very soon.

\subsubsection{The Wireless Routing Protocol}

The Wireless Routing Protocol (WRP) described in [1][12] is a table-based protocol with the goal of maintaining routing information among all nodes in the network. Each node in the network is responsible for maintaining four tables: Distance table, Routing table, Link-cost table and Message retransmission list (MRL) table

Every entry of the MRL contains the sequence number of the update message, a retransmission counter, an acknowledgmentrequired flag vector with one entry per neighbor, and a list of updates sent in the update message. The MRL records which updates in an update message need to be retransmitted and which neighbors should acknowledge the retransmission [7]. Mobiles send update messages after processing updates from neighbors or detecting a change in a link to a neighbor. In the event of the loss of a link between two nodes, the nodes send update messages to their neighbors. The neighbors then modify their distance table entries and check for new possible paths through other nodes. Part of the novelty of WRP stems from the way in which it achieves loop freedom. In WRP, routing nodes communicate the distance and second-to-last hop information for each destination in the wireless networks. WRP belongs to the class of path-finding algorithms with an important exception. It avoids the "count-to-infinity" problem [6] by forcing each node to perform consistency checks of predecessor information reported by all its neighbors. This ultimately (although not instantaneously) eliminates looping situations and provides faster route convergence when a link failure event occurs.

\subsubsection{Fisheye State Routing}

Fisheye State Routing (FSR) [34][39][45] is an improvement of GSR. The large size of update messages in GSR dissipates a considerable amount of network bandwidth. In order to overcome this problem, FSR will use a method where each updated messages would not includes information about all nodes. As an alternative, it swaps information about neighboring nodes regularly than it does about farther nodes, thus reducing the update message size. In this way, each node gets accurate information about near neighbors' and accuracy of information decreases as the distance from the node increases. Even though a node does not have accurate information about distant nodes, the packets are routed correctly because the route information becomes more and more accurate as the packet moves closer to the destination.

\subsection{Reactive (on-demand) routing}

This type of protocols finds a route on demand by flooding the network with route request packets. The main disadvantages of such algorithms are high latency time in route finding and excessive flooding can lead to network clogging. It is also called on demand routing. It is more efficient than proactive routing and most of the current work and modifications have 
been done in this type of routing for making it more and more better. The main idea behind this type of routing is to find a route between a source and destination whenever that route is needed whereas in proactive protocols we were maintaining all routes without regarding its state of use. So in reactive protocols we don't need to bother about the routes which are not being used currently. This type of routing is on demand. Discovering the route on demand avoids the cost of maintaining routes that are not being used and also controls the traffic of the network because it doesn't send excessive control messages which significantly create a large difference between proactive and reactive protocols. Time delay in reactive protocols is greater comparative to proactive types since routes are calculated when it is required. e. g. AODV (Ad-hoc On Demand Distance Vector)[32], DSR (Dynamic Source Routing)[13][31], TORA (Temporally Ordered Routing Algorithm)[33][16].

\subsubsection{Dynamic Source Routing Protocol}

The Dynamic Source Routing (DSR)[13][31] protocol is a distance-vector routing protocol for MANETs. When a node generates a packet to a certain destination and it does not have a known route to that destination, this node starts a route discovery procedure. Therefore, DSR is a reactive protocol. One advantage of DSR is that no periodic routing packets are required. DSR also has the capability to handle unidirectional links. Since DSR discovers routes on-demand, it may have poor performance in terms of control overhead in networks with high mobility and heavy traffic loads. Scalability is said to be another disadvantage of DSR [2], because DSR relies on blind broadcasts to discover routes. There are two main operations in DSR, route discovery and route maintenance. During the route discovery procedure, routers maintain ID lists of the recently seen requests to avoid repeatedly processing the same route request. Requests are discarded if they were processed recently since they are assumed to be duplicates. If a router receives a request and detects that the request contains its own ID in the list of intermediate routers, this router discards the request to avoid loops. The route maintenance procedure is used when routes become invalid due to the unpredictable movement of routers. Each router monitors the links that it uses to forward packets. Once a link is down, a route error packet is immediately sent to the initiator of the associated route. Therefore, the invalid route is quickly discarded. The initiator and all intermediate routers build routing entries associated with this new sequence number when they receive the reply. The number of hop values can be used to find a shorter path if a router receives two replies with the same destination sequence number.

\subsubsection{Ad Hoc On-demand Distance Vector Routing protocol}

AODV is reactive protocol and construct route on demand and aims to reduce routing load [3][12][32]. It uses a table driven routing framework and destination sequence numbers for routing packets to destination mobile nodes and has location independent algorithm. It sends messages only when demanded and it has bi-directional route from the source and destination. When it has packets to send from source to destinations mobile node $(\mathrm{MN})$ then it floods the network with route request (RREQ) packets. When a node receives an AODV control packet from a neighbor, or creates or updates a route for a particular destination or subnet, it checks its route table for an entry for the destination. All mobile nodes that receive the
RREQ checks its routing table to find out that if it is the destination node or if it has fresh route to the destination then it unicast route reply (RREP) which is routed back on a temporary reverse route generated by RREQ from source node, or else it re-broadcast RREQ.

\subsubsection{Temporally Ordered Routing Algorithm}

The TORA routing protocol is based on the LMR protocol [33][54]. It uses similar link reversal and route repair procedure as in LMR, and also the creation of a DAGs, which is similar to the query/reply process used in LMR[44]. Therefore, it also has the same benefits as LMR. The advantage of TORA[16] is that it has reduced the far-reaching control messages to a set of neighboring nodes, where the topology change has occurred. Another advantage of TORA is that it also supports multicasting; however this is not incorporated into its basic operation. TORA can be used in conjunction with lightweight adaptive multicast algorithm (LAM) to provide multicasting. The disadvantage of TORA is that the algorithm may also produce temporary invalid routes as in LMR.

\subsection{Zone Based Hierarchical Routing Protocols}

The Zone-Based Hierarchical Link State Protocol is based on the GPS (Global Positioning System). ZHLS is similar to the Zone Routing Protocol__It is a hybrid routing protocol acting similar like ZRP. The protocol is proactive when the destination node is in the same zone as the node which sent the request (Intrazone Clustering), here we will discuss few of them as below:

\subsubsection{The Zone Routing Protocol}

The Zone Routing Protocol (ZRP) [17][19][25] localizes the nodes into sub-networks (zones). Within each zone, proactive routing is adapted to speed up communication among neighbors. The inter-zone communication uses on-demand routing to reduce unnecessary communication. An improved mathematic model of topology management to organize the network as a forest, in which each tree is a zone, is introduced in [18]. This algorithm guarantees overlap-free zones. Furthermore, the concept introduced in this algorithm also works with QoS control because the topology model is also an approach to estimate the link quality[13]. An important issue of zone routing is to determine the size of the zone. An enhanced zone routing protocol, Independent Zone Routing (IZR), which allows adaptive and distributed reconfiguration of the optimized size of zone, is introduced in . Furthermore, the adaptive nature of the IZR enhances the scalability of the ad hoc network.

\subsubsection{The Hybrid Ad hoc Routing Protocol}

It is an improvement of the abovementioned proactive and reactive or the combination of other equipment, such as global positioning system (GPS) and other equipment, participate in the study of mechanisms to facilitate the routing of the quick search, and data transmission.[12][27]. HARP aims at establishing the most stable path from a source to a destination in order to improve delay performance due to path failure [30]. HARP applies the path discovery mechanism between zones that intends to limit flooding in the network, and that filters the 
candidate paths as soon as possible according to the stability criteria. As stability is the most desired parameter, HARP offers different mechanisms to anticipate path failure along with path maintenance procedure whose complexity is reduced by the proactive nature of the routing algorithm within a zone. These procedures reduce the delay that stems from a path failure during data transmission.

\subsubsection{The Zone-based Hierarchical Link State routing (ZHLS)}

The network is divided into zones. Each node is assumed to know its location and hence be able to map a given location to its corresponding zone id. Two zones are assumed to be connected if at least one node in one zone is connected to a node in the other zone. Routing within and in between zones is based on shortest path routing. Hence, ZHLS [5][25][30] belongs to the category of routing protocols based on minimum-weight path based routing.

\subsection{Cluster-based Routing Protocols}

Cluster Based Routing Protocol (CBRP) is an on-demand routing protocol, where the nodes are divided into clusters. In this section we will discuss few typical type of protocol based on CBRP.

\subsubsection{The Cluster head Gateway Switch Routing (CGSR)}

Nodes are grouped into clusters and a cluster head controls the cluster. One of the important criteria for cluster-head election algorithms is stability. Frequent cluster head election can result in prohibitive overhead. In CGSR [21], a stable least cluster change (LCC) clustering algorithm is preferred over the widely used lowest (highest) ID and the highest connectivity algorithms. According to the LCC algorithm, cluster heads change only when two cluster heads come into contact, or a node moves out of the range of all cluster heads. At each mobile node, a "cluster member-table" is maintained where in information about the destination cluster head of each mobile node in the network is stored. In addition, a routing table that stores information about the next hop to reach the destination is stored at each node. On receiving a packet, a node uses the cluster member table to determine the nearest cluster head along the route to the destination; then uses the routing table to determine the next hop node used to reach the selected cluster head. Using DSDV[5][28], the cluster member table is periodically exchanged among all nodes in the network and the routing table is periodically exchanged within a cluster. Traffic from a source to destination is routed using a hierarchical cluster head-gateway routing approach where DSDV is the underlying routing scheme. CGSR fits under the minimumweight path routing category.

\subsubsection{The Hierarchical State Routing (HSR)}

The characteristic feature of Hierarchical State Routing (HSR) [20] is multilevel clustering and logical partitioning of mobile nodes. The network is partitioned into clusters and a cluster-head elected as in a cluster-based algorithm. In HSR, the cluster-heads again organize themselves into clusters and so on. Hierarchical state routing (HSR), proposed in Scalable Routing Strategies for Ad Hoc Wireless Network [15][18], is a typical example of a hierarchical routing protocol. HSR maintains a hierarchical topology, where elected clusterheads at the lowest level become members of the next higher level. On the higher level, superclusters are formed, and so on. Nodes which want to communicate to a node outside of their cluster ask their clusterhead to forward their packet to the next level, until a clusterhead of the other node is in the same cluster. The packet then travels down to the destination node. Furthermore, HSR proposes to cluster nodes in a logical way instead of in a geological way: members of the same company or in the same battlegroup are clustered together, assuming they will communicate much within the logical cluster. HSR does not specify how a cluster is to be formed.

\subsubsection{Cluster Based Routing Protocol (CBRP)}

The network is divided into clusters. Cluster heads are elected using the "min-ID" algorithm. Route discovery in CBR [37] is similar to that in DSR except that the forwarding nodes of the route discovery packets are only the cluster heads and gateways. Route shortening is done if two gateways or cluster heads can directly reach each other without one or more intermediate nodes on the route. Thus, CBR is designed to aim for the shortest hop route from the source to the destination across one or more intermediate clusters. CBR could be grouped under the category of routing protocols based on the minimum-weight path routing.

\subsection{Routing Protocols using Location Information}

\subsubsection{Location Aided Routing (LAR)}

Location Aided Routing (LAR) [43] is another kind of hybrid routing protocol. LAR is a scalable routing protocol that uses landmarks, location and distance of the nodes to reduce the periodical update costs. LAR is suitable for networks with large number of nodes, which need to establish a hierarchy. This protocol is more complex than zone routing protocols due to the fact that the maintenance of hierarchical network is more difficult when determining the level of the nodes in the hierarchy. Some research effort has been put on the adaptation of classic ad hoc routing protocols [12][18], such as DSR and AODV, to the scalable networks. The possibility of applying the DSR and AODV to scalable networks is studied and an improvement of DSR and AODV is presented in order to apply them to scalable networks [2].

\subsubsection{The Distance Routing Effect Algorithm for Mobility}

DREAM [23] is a proactive, multi-path, location-aware routing protocol. DREAM makes use of the so called distance effect to regulate the frequency of topological updates. According to the distance effect, the greater the distance between two nodes, the lower is their relative mobility. DREAM also makes use of the mobility rate of the nodes to regulate the frequency of location updates: the faster a node moves, the higher is the frequency of 
location updates from that node. A node records the locations of all its peer nodes in a location table. Using this location information, a node forwards the data packet to a set of neighbors that lie in the direction to the destination. If no such neighbors could be selected, the data packet is dropped. The destination responds with an ACK when it receives the data packet forwarded by a designated set of nodes.

The ACK is forwarded to the source node in a fashion similar to that of the data packet. If the source node fails to receive an ACK through a designated set of nodes, it floods the data packet. Once at least one path between the source and destination are learnt, the source could start sending data packets using the learned paths, preferably the shortest hop path. The routing metric in DREAM has been referred to as shortest hop path in [5]. Hence, DREAM belongs to the class of protocols based on minimum-weight path based routing.

\subsection{Link Stability Based Routing Protocols}

\subsubsection{The Associatively Based Routing Protocol}

The Associatively Based Routing (ABR) protocol [41][42] is another source initiated routing protocol, which also uses a query-reply technique to determine routes to the required destinations. However, in ABR route selection is primarily based on stability. To select stable route each node maintains an associatively tick with their neighbors, and the links with higher associatively tick are selected in preference to the once with lower associatively tick. However, although this may not lead to the shortest path to the destination, the routes tend to last longer. Therefore, fewer route reconstructions are needed, and more bandwidth will be available for data transmission. The disadvantage of $\mathrm{ABR}$ is that it requires periodic beaconing to determine the degree of associatively of the links. This beaconing requirement requires all nodes to stay active at all time, which may result in additional power consumption. Another disadvantage is that it does not maintain multiple routes or a route cache, which means that alternate routes will not be immediately available, and a route discovery will be required using link failure. However, ABR has to some degree compensated for not having multiple routes by initiating a localized route discovery procedure (i.e. LBQ).

\subsubsection{The Signal Stability-Based Adaptive Routing protocol (SSR)}

The Signal Stability-Based Adaptive (SSA) routing protocol [14] selects routes based on the signal strength between nodes. Signal strength of the link with a neighboring node is determined using the periodic beacons received from that node. If the signal strength is beyond a threshold, the link is considered stable; otherwise, the link is designated to be weak. Preference is given to paths on the stronger stable channels, SSA fits under the stability category. Route discovery in SSA is through source-initiated broadcast request messages. A node forwards the request message to the next hop only if it is received over a stronger channel and has not been previously processed. The destination, unlike in ABR, chooses the first arriving route-search packet and sends back a route-reply in the reverse direction of the selected route. In addition to choosing the path of strongest signal stability, it is most likely that first arriving route-search packet traversed over the shortest and/or the least congested path. If no route-reply message is received within a specific timeout period, the source initiates another route-search and also indicates its acceptability of weak channels in the search packet header.

\section{COMPARISONS AND ANALYSIS OF ROUTING PROTOCOLS}

This section presents over all comparison of MANET routing protocols, first generalized DSDV, WRP, FSR, DSR, ZRP, AODV, TORA, CGSR, ZRP, SSR, and ABR protocols after that analysis for same groups as discussed above. We have presented the comparison among routing protocols show in table 1, table 2, and table 3 the kind of protocols such as WRP, DSDV and FSR proactive, DSR, AODV, TORA, SSR and $A B R$ are reactive where as $\mathrm{ZRP}$ is zone-based routing and CGSR is cluster-based routing. Routing structure of CGSR is hierarchical and all other have flat, all these protocols are loop free only WRP is loop free but not instantaneous. As reactive routing protocols for mobile ad hoc networks, DSR, AODV and TORA are proposed to reduce the control traffic overhead and improve scalability. WRP, DSDV and FSR have distinct features and use different mechanisms for loop-free guarantee. WRP, DSDV and FSR have the same time and communication complexity. Both DSR and TORA support unidirectional links and multiple routing paths, but AODV doesn't. In contrast to DSR and TORA, nodes using AODV periodically exchange hello messages with their neighbors to monitor link disconnections. WRP, FSR and TORA have characteristic as reduced topology and all other have full topology, only. AODV and ZRP have multicasting capability other have no such capability. As shown in table 3: each protocol has advantages and disadvantages. No any protocol which out perform in all condition. Comparisons shown in table 1, table 2 and table 3 based on basic characteristics of routing protocols have clearly defined and all categories [46] in well mannered.

The proactive routing in mobile ad hoc networks needs mechanisms that dynamically collect network topology changes and send routing updates in an event-triggered style. Protocols WRP, DSDV and FSR are loop free and have the same time and communication complexity. Whereas WRP has a large storage complexity compared to DSDV because more information is required in WRP to guarantee reliable transmission and loop-free paths. Both periodic and triggered updates are utilized in WRP and DSDV; therefore, their performance is tightly related with the network size and node mobility pattern. As a Link State routing protocol, FSR has high storage complexity, but it has potentiality to support multiple-path routing and QoS routing. 
Table 1: Comparison of Basic characteristics of routing protocols

\begin{tabular}{|c|c|c|c|c|c|}
\hline Protocol & Routing Category & $\begin{array}{l}\text { Routing } \\
\text { Structure }\end{array}$ & Loop free & Route Metric & $\begin{array}{l}\text { Power } \\
\text { requirement }\end{array}$ \\
\hline DSDV & Proactive & Flat & Yes & Shortest path & High \\
\hline WRP & Proactive & Flat & $\begin{array}{c}\text { Yes, but not } \\
\text { instantaneou } \\
\text { s }\end{array}$ & Shortest path & High \\
\hline FSR & Proactive & Flat & Yes & Shortest path & High \\
\hline DSR & Reactive & Flat & Yes & $\begin{array}{c}\text { Shortest path or } \\
\text { next available in } \mathrm{RC}\end{array}$ & Low \\
\hline AODV & Reactive & Flat & Yes & $\begin{array}{c}\text { Fastest and Shortest } \\
\text { path }\end{array}$ & Low \\
\hline TORA & Reactive & Flat & Yes & Shortest path & Low \\
\hline ZRP & Zone-based routing & Flat & Yes & Shortest path & Medium \\
\hline CGSR & $\begin{array}{c}\text { Cluster-based } \\
\text { routing }\end{array}$ & Hierarchical & Yes & Shortest path & High \\
\hline SSR & Reactive & Flat & Yes & Signal \& Stability & Low \\
\hline ABR & Reactive & Flat & Yes & $\begin{array}{c}\text { Link Associatively \& } \\
\text { shortest path\& } \\
\text { others }\end{array}$ & Low \\
\hline
\end{tabular}

As reactive routing protocols for mobile ad hoc networks, DSR, AODV and TORA are proposed to reduce the control traffic overhead and improve scalability. Both DSR and TORA support unidirectional links and multiple routing paths, but AODV doesn't. TORA, utilizing the "link reversal" algorithm, DAG constructs routing paths from multiple sources to one destination and supports multiple routes and multicast [2]. In AODV and DSR, a node notifies the source to re-initiate a new route discovery operation when a routing path disconnection is detected. In TORA, a node re-constructs DAG when it lost all downstream links. AODV uses sequence numbers to avoid formation of route loops. Because DSR is based on source routing, a loop can be avoided by checking addresses in route record field of data packets. In TORA, each node in an active route has a unique height and packets are forwarded from a node with higher height to a lower one.
As zone based mobile ad hoc network routing protocols, ZRP, HARP and ZHLS use different zone construction methods, which have critical effect on their performance. In ZRP, the network is separated into overlapping zones according to the topology knowledge for neighboring nodes of each node. In HARP, the network is separated into non-overlapping zones dynamically by DDR through mapping the network topology to a forest. ZHLS assumes that each node has a location system such as GPS and the geographical information is well known, and the network is geographically divided into non-overlapping zones. However, because zones heavily overlap, ZRP in general will incur more overhead than ZHLS and HARP.

Different clustering algorithms have been introduced to group mobile nodes and elect clusterheads in cluster based routing protocols [1]. A location management mechanism is used in HSR to map the logical address to the physical address. CGSR is based on DSDV, a proactive routing protocol for mobile ad hoc networks, and every node keeps routing information for other nodes in both the cluster member table and the routing table. In CBRP, every node keeps information about its neighbors and a clusterhead maintains information about its members and its neighboring clusterheads. 
Table 2: Comparison of Basic characteristics of routing protocols

\begin{tabular}{|c|c|c|c|c|c|}
\hline Protocol & Topology & $\begin{array}{c}\text { Hallow } \\
\text { Message }\end{array}$ & $\begin{array}{c}\text { Multicasting } \\
\text { Capability }\end{array}$ & Update Period & $\begin{array}{c}\text { Control } \\
\text { overhead }\end{array}$ \\
\hline DSDV & Full & No & No & Hybrid & High \\
\hline WRP & Reduced & Yes & No & Hybrid & High \\
\hline FSR & Reduced & No & No & Periodically & Low \\
\hline DSR & Full & No & No & Event driven & Low \\
\hline AODV & Full & Yes & Yes & Event driven & Low \\
\hline TORA & Reduced & No & No & Event driven & Low \\
\hline ZRP & & Yes & Yes & Periodically & Medium \\
\hline CGSR & Full & No & No & Periodically & High \\
\hline SSR & & Yes & No & Periodically/ Event driven & Low \\
\hline ABR & Full & Yes & No & Periodically/ Event driven & Low \\
\hline
\end{tabular}

Table 3: Comparison of Basic characteristics of routing protocols

\begin{tabular}{|c|c|c|c|c|}
\hline Protocol & Routing & $\begin{array}{c}\text { Multiple } \\
\text { routes }\end{array}$ & Advantages & Disadvantages \\
\hline DSDV & Uniform & No & $\begin{array}{l}\text { Freedom of loops in routing } \\
\text { tables }\end{array}$ & High overhead \\
\hline WRP & Uniform & No & $\begin{array}{l}\text { Freedom of loops in routing } \\
\text { tables }\end{array}$ & $\begin{array}{l}\text { A large amount of memory and } \\
\text { periodic hello message consumes } \\
\text { power and bandwidth }\end{array}$ \\
\hline FSR & Uniform & May be & Reduces control overhead & $\begin{array}{l}\text { High Memory overhead, reduced } \\
\text { accuracy }\end{array}$ \\
\hline DSR & Uniform & Yes & $\begin{array}{l}\text { No periodic hello message and } \\
\text { fast recovery - cache can store } \\
\text { multiple paths to a destination }\end{array}$ & $\begin{array}{l}\text { Major scalability problem due to the } \\
\text { nature of source routing and flooding, } \\
\text { large delays }\end{array}$ \\
\hline AODV & Uniform & No & $\begin{array}{l}\text { Uses bandwidth efficiently, is } \\
\text { responsive to changes in } \\
\text { topology, is scalable and } \\
\text { ensures loop free routing }\end{array}$ & $\begin{array}{l}\text { Nodes use the routing caches to reply } \\
\text { to route queries. Results } \\
\text { "uncontrolled" replies and repetitive } \\
\text { updates in hosts' caches yet early } \\
\text { queries cannot stop the propagation of } \\
\text { all query messages which are flooded } \\
\text { all over the network }\end{array}$ \\
\hline TORA & Uniform & Yes & $\begin{array}{l}\text { Provides loop free paths at all } \\
\text { instants and multiple routes so } \\
\text { that if one path is not available, } \\
\text { other is readily available. }\end{array}$ & $\begin{array}{l}\text { Temporary routing loops, } \\
\text { problem in distance vector routing } \\
\text { protocols. }\end{array}$ \\
\hline
\end{tabular}




\begin{tabular}{|c|c|c|c|c|}
\hline ZRP & Nonuniforrn & No & Reduce retransmissions & Overlapping zones \\
\hline ZHLS & Nonuniforrn & $\begin{array}{l}\text { Yes, if } \\
\text { more than } \\
\text { one virtual } \\
\text { link exists }\end{array}$ & $\begin{array}{l}\text { Reduction of SPF, low control } \\
\text { overhead }\end{array}$ & Static zone map required \\
\hline CGSR & Nonuniforrn & No & Reduced control overhead & $\begin{array}{l}\text { Too frequent cluster head selection can } \\
\text { be an overhead and cluster nodes and } \\
\text { Gateway can be a bottleneck }\end{array}$ \\
\hline HSR & Nonuniforrn & No & Low control overhead & Location management \\
\hline CBRP & Nonuniforrn & No & $\begin{array}{l}\text { Only cluster-heads } \\
\text { exchangerouting information }\end{array}$ & Cluster maintenance, temporary loops \\
\hline LAR & Nonuniforrn & Yes & Localized route discovery & $\begin{array}{l}\text { Based on source routing, flooding is } \\
\text { used if no location information is } \\
\text { available }\end{array}$ \\
\hline DREAM & Nonuniforrn & & $\begin{array}{l}\text { Low control overhead and } \\
\text { memory overhead; }\end{array}$ & Requires a GPS \\
\hline SSR & Uniform & No & $\begin{array}{l}\text { Route stability ,to select strong } \\
\text { connection leads to fewer route } \\
\text { reconstruction }\end{array}$ & $\begin{array}{l}\text { Long delay since intermediate nodes } \\
\text { can't answer the path (unlike AODV, } \\
\text { DSR) }\end{array}$ \\
\hline ABR & Uniform & Yes & $\begin{array}{l}\text { Route stability(free from } \\
\text { duplicate packets) }\end{array}$ & $\begin{array}{l}\text { Scalability problems, Short beaconing } \\
\text { interval to reflect association degree } \\
\text { precisely }\end{array}$ \\
\hline
\end{tabular}

Location based routing protocols exploit location and node mobility information for the routing process. LAR, DREAM and GLS use the information in different ways and provide different services. LAR can be integrated into a reactive routing protocol and its main objective is to perform more efficient route discovery and limit the flooding of route request packets. In DREAM, the location update frequency is determined by the relative distance between nodes and their mobility characteristics. GLS is not a routing protocol, but only provides a location service. In GLS, every node has several location servers scattered throughout the network which provide location information.

In mobile ad hoc networks, node mobility causes link state changes and results in route maintenance operations [41][42]. Using stability of links instead of hop numbers as metric for routing path selection is a promising solution for reducing control overhead. Although ABR and SSR are all based on Link State routing algorithm, they have distinct features and different mechanisms. ABR is a reactive routing protocol and is proposed to incorporate the link stability into routing to construct long-lived routing paths. The metric associatively is used in ABR to measure how long a wireless link lasts without failure. Following the assumption that the number of the associatively tags of a link reflects how long the link will be available in the future, a route path with greatest associatively tags is constructed. SSR can be seen as an extension of ABR. SSR uses signal stability as routing metric and route requests are propagated only through strong channels. SSR also assumes that the current signal strength of a channel can be used to predict its state in the future. Additionally, in SSR the messages are only propagated through strong channels to reduce the traffic overhead.

\section{APPLICATIONS AND CHALLENGES}

\subsection{Applications of Mobile Ad-hoc Networks}

Ad hoc wireless networks have an important role to play in military applications [5][15]. Soldiers equipped with multimode mobile communicators can now communicate in an ad hoc manner without the need for fixed wireless base stations. In addition, small vehicular devices equipped with audio sensors and cameras can be deployed at targeted regions to collect important location and environmental information which will be communicated back to a processing node via ad hoc mobile communications.

People today attend conferences and meetings with their palmtops, laptops, and notebooks. It is therefore attractive to have instant network formation, in addition to file and information sharing without the presence of fixed base stations and systems administrators. Presenters can multicast slides and audio to intended recipients. Attendees can ask questions and interact on a commonly shared whiteboard. Ad hoc mobile communication is particularly useful in relaying information (status, situation awareness, etc.) via data, video, and/or voice from one rescue team member to another over a small handheld or wearable wireless device. Again, this applies to law enforcement personnel as well. Applications of mobile ad-hoc networks are tabulated as shown in table 4: 
Table 4. Application for the Ad Hoc Networks.

\begin{tabular}{|c|c|}
\hline Applications & The Possible Service of Ad Hoc Networks \\
\hline Emergency services & $\begin{array}{ll} & \text { Search and rescue operations in the desert and in the mountain and so on. } \\
\text { - } & \text { Replacement of fixed infrastructure in case of environmental disasters } \\
\text { - } & \text { Policing } \\
\text { - } & \text { Fire fighting } \\
& \text { Supporting doctors and nurses in hospitals }\end{array}$ \\
\hline Education & $\begin{array}{l}\text { - Universities and campus settings } \\
\text { - } \quad \text { Classrooms } \\
\text { - } \\
\end{array}$ \\
\hline $\begin{array}{l}\text { Context aware } \\
\text { services }\end{array}$ & $\begin{array}{l}\text { - Follow-on services: call-forwarding, mobile workspace } \\
\text { - } \quad \text { Information services: location specific services, time dependent services } \\
\text { - Infotainment: touristic information }\end{array}$ \\
\hline Tactical networks & $\begin{array}{l}\text { - } \quad \text { Military communication. } \\
\text { - } \quad \text { Military operations in the battlefields }\end{array}$ \\
\hline Coverage extension & $\begin{array}{l}\text { - Extending cellular network access } \\
\text { - } \quad \text { Linking up with the internet, intranets, and so on. }\end{array}$ \\
\hline Sensor networks & $\begin{array}{l}\text { - Inside the home: smart sensors and actuators embedded in consumer electronics. } \\
\text { - } \quad \text { Body area networks (BAN) } \\
\text { - } \quad \text { chemical/biological detection }\end{array}$ \\
\hline $\begin{array}{l}\text { Home and enterprise } \\
\text { networks }\end{array}$ & $\begin{array}{l}\text { - Using the wireless networking in Home or office. } \\
\text { - } \quad \text { Conferences, meeting rooms } \\
\text { - } \quad \text { Pheme parks } \\
\end{array}$ \\
\hline $\begin{array}{l}\text { Commercial and } \\
\text { civilian } \\
\text { environments }\end{array}$ & $\begin{array}{l}\text { - } \quad \text { E-commerce: electronic payments anytime and anywhere } \\
\text { - } \quad \text { Business: dynamic database access, mobile offices } \\
\text { Vehicular services: road or accident guidance, transmission of road and weather conditions, } \\
\text { taxi cab network, inter-vehicle networks } \\
\text { - Sports stadiums, trade fairs, shopping malls and so on. } \\
\text { Networks of visitors inside the airports. }\end{array}$ \\
\hline
\end{tabular}

\subsection{Real Challenges for Mobile Ad-hoc Networks}

Ad hoc networks have to suffer many challenges at the time of routing[36]. Dynamically changing topology and no centralized infrastructure are the biggest challenges in the designing of routing protocols in mobile ad hoc network. The position of the nodes in an ad hoc network continuously varies due to which we can't say that any particular protocol will give the best performance in each and every case topology varies very frequently so we have to select a protocol which dynamically adapts the ever-changing topology very easily.

Another challenge in MANET is limited bandwidth. If we compare it to the wired network then wireless network has less and more varying bandwidth. So bandwidth efficiency is also a major concern in ad-hoc routing protocol designing because sometimes data has to be transmitted within real time constraints. Limited power supply is the biggest challenge of an Ad hoc network so if we want to increase the network lifetime (duration of time when the first node of the network runs out of energy)[11] as well the node lifetime then we must have an energy efficient protocol. So an ad hoc routing protocol must meet all these challenges to give the average performance in every case. The few other current challenges of mobile ad-hoc networks are listed as:

- Multicast

- QoS support

- Limited wireless transmission range

- Broadcast nature of the wireless medium

- Packet losses due to transmission errors

- Mobility-induced route changes

- Mobility-induced packet losses

- Battery constraints

- $\quad$ Potentially frequent network partitions

- $\quad$ Ease of snooping on wireless transmissions(security hazard)

\section{CONCLUSIONS DIRECTIONS}

\subsection{Conclusions}

We presented a comprehensive survey of the routing protocols for mobile ad hoc wireless networks. We discussed the common goals of the methodologies of a routing protocol is to reduce control packet overhead, minimize the end-to-end delay, and maximize throughput; however, they differ in ways of finding and/or maintaining the routes between source- 
destination pairs. To the best of our knowledge, we could not find such a comprehensive survey on MANET routing protocols in the literature. This paper first present methodology of the typical types of routing protocols and then compared these protocols based on common characteristics. After that we presented applications and real challenges of routing in MANET. We believe our survey will be very helpful to the research community and also serve as a huge introductory material for somebody embarking onto research in routing protocols in ad hoc wireless networks. From technological view of point

This paper can support formal verification of MANET routing protocols or characterization of these protocols can aid the design, comparison, and improvement of these protocols with incorporating others good feature.

\subsection{Future Directions}

Mobile ad-hoc networks have received increasing research attention in recent years. There are various active research works with MANETs focuses on promising future research directions based on the current research. This suggests a potential research topic on MANET routing in which estimates of parameters, including network and traffic profiles, can be used to adaptively choose different routing protocols or different modules for one protocol.

Further study of node mobility is also a promising research direction to improve estimates of link and path lifetimes, and improve the performance of MANET routing protocols. More extensive simulation and emulation studies can be used to analyze and to guide users when they choose routing protocols for their MANET applications and aid designers in improving protocols. A framework that characterizes these protocols can aid the design, comparison, and improvement of these protocols. Analysis and conclusions can guide users when they choose routing protocols for their MANET applications and aid designers in improving protocols.

\section{REFERENCES}

[1] T. Lin, S. F. Midkiff, and J. S. Park, "A Framework for Wireless Ad Hoc Routing Protocols," in Proc. of IEEE Wireless Communications and Networking Conference (WCNC), vol. 2, pp. 1162-1167, 2003.

[2] Shiva Prakash, J.P. Saini, and S.C. Gupta, "Performance Analysis of Routing Protocols in Wireless Ad-hoc Network", International journal of computer information systems (ISSN: 2229-5208), Silicon Valley Publishers, October, 2010

[3] Nor Surayati Mohamad Usop, Azizol Abdullah, "Performance Evaluation of AODV, DSDV \& DSR Routing Protocol in Grid Environment", IJCSNS International Journal of Computer Science and Network Security, 9, No.7, July 2009

[4] J. Wells, "A Network Mobility Survey and Comparison with a Mobile IP Multiple Home Address Extension," M.S. thesis, The Bradley Department of Electrical and Computer Engineering, School of Engineering, Virginia Tech, USA, 2003. Available at http://scholar.lib.vt.edu/theses/available/etd-12272003225555.

[5] Saleh Ali K. Al-Omari and Putra Sumari, " An over view of Mobile Ad Hoc Networks for Existing Protocols and
Applications," International Journal on applications of graph theory in wireless ad hoc networks and sensor networks (Graph-Hoc), Vol.2, March 2010.

[6] J. Moy, "OSPF Version 2," Internet Engineering Task Force RFC 2328, April 1998. Available at http://www.ietf.org/rfc/rfc2328.txt.

[7] Zhi Li and Yu-Kwong Kwok, "A New Multipath Routing Approach to Enhancing TCP Security in Ad Hoc Wireless Networks," Proc. ICPP Workshops, pp. 372379, June 2005.

[8] Peng Yang and Biao Huang, "Multi-path Routing Protocol for Mobile Ad Hoc Network," 2008 International Conference on Computer Science and Software Engineering

[9] A. Tsirigos and Z. J. Haas, "Analysis of Multipath Routing-Part I: The Effect on the Packet Delivery Ratio," IEEE Trans. Wireless Communications, vol. 3, no. 1, pp. 138-146, Jan. 2004.

[10] Benamar KADRI, Mohammed FEHAM and Abdallah M'HAMED, "Weight based DSR for Mobile Ad Hoc Networks," in 3rd International Conference on Information and Communication Technologies: From Theory to Applications, 2008. ICTTA 2008. pp. 1- 6, 7 11 April 2008.

[11] Jangsu Lee, Seunghwan Yoo, and Sungchun Kim, "Energy aware Routing in Location based Ad-hoc Networks," Proceedings of the 4th International Symposium on Communications, Control and Signal Processing, ISCCSP 2010, Limassol, Cyprus, 3-5 March 2010

[12] Samba Essay, Zongkai Vang, Jianhua He, "A survey on mobile ad hoc wireless network", Asian Network for Scientific Information, White Paper http://whitepapers.zdnet.com/ whitepaper.aspx?\&docid=148894\&promo $\quad=100511$, 2004.

[13] D. Maltz, Y. Hu, "The Dynamic Source Routing Protocol for Mobile Ad Hoc Networks", Internet Draft, July 2004, Available: http://www.ietf.org/internet-drafts/draft-ietfmanet-dsr-10.txt

[14] R. Dube et al., Signal stability based adaptive routing for ad hoc mobile networks, IEEE Pers. Comm., February 1997, p 36-p45.

[15] B. B. Maqbool and M. A. Peer, "Classification of Current Routing Protocols for Ad Hoc Networks - A Review", International Journal of Computer Applications (0975 - 8887), Volume 7- No.8, October 2010

[16] V. Park, and S. Corson, Temporally-Ordered Routing Algorithm (TORA) Version 1 Functional Specification. IETF Internet draft, 1997.

[17] J. Schaumann, "Analysis of the Zone Routing Protocol", December 2002.

[18] M. Abdoos, K. Faez, and M. Sabaei, "Position Based Routing Protocol with More Reliability in Mobile Ad Hoc Network", World Academy of Science, Engineering and Technology,49 2,009

[19] Haas ZJ, Pearlman MR, Samar P, "The Zone Routing Protocol (ZRP) for Ad Hoc Networks", IETF draft, July 
2002, available at http://tools.ietf.org/id/draft-ietfmanetzone-zrp-04.txt. 21 February 2008.

[20] A. Iwata, C.-C. Chiang, G. Pei, M. Gerla, and T.-W. Chen, Scalable routing strategies for ad hoc wireless networks. IEEE Journal on Selected Areas in Communications, Special Issue on Ad-Hoc Networks, August 1999, p1369-p1379.

[21] C. C. Chiang, T. C. Tsai, W. Liu and M. Gerla, Routing in clustered multihop, mobile wireless networks with fading channel, The Next Millennium, The IEEE SICON, 1997.

[22] B. Bellur, et. al, "Topology Dissemination Based on Reverse-Path Forwarding (TBRPF)", IETF Internet Draft, draft-ietf-manet-tbrpf-08.txt, April 2003.

[23] Stefano Basagni, Irnich Chlamtac, Violet R. Syrotiuk and Barry A. Woodward, "A Distance Routing Effect Algorithm for Mobility (DREAM)," in Proceedings of the 4th annual ACM/IEEE international conference on Mobile computing and networking texas, United States, pages :76-84 in 1998.

[24] M. Abolhasan, B. Hagelstein, J. C.P. Wang, "Real-world performance of current proactive multi-hop mesh protocols", IEEE APCC, Shanghai, China, 8-10th October 2009.

[25] Haas ZJ, Pearlman MR, Samar P, "The Zone Routing Protocol (ZRP) for Ad Hoc Networks", IETF draft, July 2002, available at http://tools.ietf.org/id/draft-ietfmanetzone-zrp-04.txt. Accessed 21 February 200894 A.-S.K. Pathan and C.S. Hong

[26] D. D. Perkins, H. D. Hughes, and C. B. Owen, "Factors affecting the performance of ad hoc networks," in Proc. of IEEE International Conference on Communications (ICC) 2002, vol. 4, pp. 2048-2052, 2002. J.

[27] Mehran Abolhasan , Tadeusz Wysocki, and Eryk Dutkiewic, "A review of routing protocols for mobile ad hoc networks," Ad Hoc Networks 2 (2004) 1-22, www.elsevier.com/locate/adhoc

[28] A. Boukerche, "Performance comparison and analysis of ad hoc routing algorithms," in Proc. of IEEE International Conference on Performance, Computing, and Communications, pp. 171-178, 2001.

[29] B. Bellur, R. G. Ogier, and F. L. Temlin," Topology Dissemination Based on Reverse-Path Forwarding (TBRPF)", RFC 3684, February 2004.

[30] N. Nikanein, C. Bonnet, and N. Nikaein, "Hybrid Ad Hoc Routing Protocol - HARP," in proceeding of IST 2001: International Symposium on Telecommunications http://www.eurecom.fr/ nikaeinn/harp.ps

[31] D. B. Johnson, D. A. Maltz, Y. C. Hu, and J. G. Jetcheva, "The Dynamic Source Routing Protocol for Mobile Ad Hoc Networks (DSR)," Internet Engineering Task Force 208 (IETF) draft, Febuary 2002.

[32] C. E. Perkins, E. M. Belding-Royer, and S. R. Das, "Ad hoc On-Demand Distance Vector (AODV) Routing," Internet Engineering Task Force (IETF) draft, November 2002.
[33] V. Park and S. Corson, "Temporally-Ordered Routing Algorithm (TORA) Version 1 Functional Specification," Internet Engineering Task Force (IETF) draft, July 2001.

[34] M. Gerla, X. Y. Hong, and G. Y. Pei, "Fisheye State Routing Protocol (FSR) for Ad Hoc Networks," Internet Engineering Task Force (IETF) draft, June 2001.

[35] Z. J. Haas, M. R. Pearlman, and P. Samar, "The Intrazone Routing Protocol (IARP) for $\mathrm{Ad} \mathrm{Hoc}$ Networks," Internet Engineering Task Force (IETF) draft, July 2002.

[36] C. Ying, Q. Lv, Y. Liu, and M. Shi, "Routing protocols overview and design issues for self-organized network," in Proc. of International Conference on Communication Technology, vol. 2, pp. 1298-1303, 2000.

[37] M. Jiang, J. Li, Y. C. Tay, "Cluster Based Routing Protocol (CBRP) Functional Specification Internet Draft," http://tools.ietf.org/html/draft-ietf-manet-cbrpspec, work in progress, June 1999.

[38] T. Camp, J. Boleng, and V. Davies, "A survey of mobility models for ad hoc network research," in Wireless Communication \& Mobile Computing (WCMC): Special issue on Mobile Ad Hoc Networking: Research, Trends and Applications, vol. 2, no. 5, pp. 483-502, 2002.

[39] G. Pei, M. Gerla, and T.W. Chen, "Fisheye state routing: a routing scheme for ad hoc wireless networks," in Proc. of IEEE International Conference on Communications, pp. 70-74, 2000.

[40] INRIA “Optimized Link State Routing," December 2001. Available at http://menetou.inria.fr/olsr.

[41] Hai-Keong Toh, "Associativity-Based Routing for AdHoc Mobile Networks in Wireless Personal Communications," An International Journal Volume 4, Issue 2 (March 1997) Pages: 103 - 139.

[42] Tai Hieng Tie, Chong Eng Tan and Sei Ping Lau, "Alternate Link Maximum Energy Level Ad-hoc Distance Vector Scheme for Energy Efficient Ad-hoc Networks Routing," in International Conference on Computer and Communication Engineering (ICCCE 2010), 11-13 may 2010, Kuala Lumpur, Malaysia.

[43] Young-Bae Ko and Nitin H. Vaidya, "Location-Aided Routing (LAR) in mobile ad-hoc networks in Wireless Networks," Volume 6, Pages: 307 - 321 Issue 4 (July 2000).

[44] M.S. CORSON AND A. EPHREMIDES, "Lightweight Mobile Routing protocol (LMR), A distributed routing algorithm for mobile wireless networks, Wireless Networks 1 (1995).

[45] M. Geria, Guangyu, X. Hong, and T. Chen, "Fisheye State Routing Protocol (FSR) for Ad Hoc Networks Internet Draft," http://tools.ietf.org/html/draft-ietf-manetfsr, work in progress, June 2001.

[46] Shiva Prakash, J.P. Saini, Sandip Vijay, and S.C. Gupta, "Performance Analysis of Routing Protocols in Wireless Ad-hoc Network", International journal of computer information systems (ISSN: 2229-5208), Silicon Valley Publishers, October, 2010. 